Pacific Journal of Mathematics

of 


\title{
ALMOST SMOOTH PERTURBATIONS OF SELF-ADJOINT OPERATORS
}

JoHN B. Butler, JR.

\begin{abstract}
Assume $H^{0} \in \mathscr{C}(\mathfrak{S})$ is a self-adjoint operator with spectrum on $[0, \infty)$ and that $E^{0}(\Delta) \in \mathscr{B}(\mathfrak{H})$ is the spectral measure determined by $H^{0}, \Delta \subset[0, \infty)$. Let $H^{1}=H^{0}+V$ where $V=B \cdot A$ and $A, B \in \mathscr{B}(\mathfrak{S})$ are commuting self-adjoint operators. In this paper T. Kato's concept of smooth perturbations is generalized in the following way: $H^{1}$ is said to be an almost smooth perturbation of $H^{0}$, except at $1=0$, if $A, B$ are smooth with respect to $H^{0} E^{0}\left(\Delta_{m}\right)$ for all intervals $\Delta_{m}=(1 / m, \infty), m \geqq 1$. It is proved that the time independent wave operators corresponding to $H^{0}, H^{1}$ exist when the assumption that $H^{1}$ is smooth with respect to $H^{0}$ is replaced by the assumption that $H^{1}$ is almost smooth with respect to $H^{0}$.
\end{abstract}

The concept of smooth perturbations was introduced by T. Kato in [2]. The importance of the generalization given here is that it allows one to apply the theory developed in [2] to certain one dimensional differential operators which are almost smooth but not smooth. Examples of some almost smooth ordinary differential operators are given below in $\S 3$.

2. The wave operators. Let $\Omega_{ \pm}$denote the upper and lower complex plane, with the reals excluded, and let $f$ be a function on $\Omega_{ \pm} \times \mathfrak{F}$ into $\mathfrak{S}$. Such a function $f$ is said to be in the Hardy class $H_{2}((-\infty, \infty): \mathfrak{S})$ if and only if $f$ is analytic in $\lambda$ for all $\lambda \in \Omega_{ \pm}$and for all $u \in \mathfrak{S}, \delta>0, \int_{-\infty}^{\infty}\|f(1 \pm i \delta ; u)\|^{2} d 1 \leqq P\|u\|^{2}$ for some $P>0$ independent of $u$ and $\delta$. An operator $A \in \mathscr{B}(\mathscr{S})$ is said to be smooth with respect to $H^{0}$ if and only if the function $f$ defined by

$$
(\lambda, u) \mapsto A\left(H^{0}-\lambda I\right)^{-1} u=A R^{0}(\lambda) u \text { is in } H_{2}((-\infty, \infty): \mathfrak{F})
$$

[2, p. 260]. ${ }^{1)}$ Now we shall make the following assumptions regarding $H^{0}, A, B$ :

(i) For some $N$, $\left\|B R^{0}(\lambda) E^{0}\left(A_{m}\right) A\right\| \leqq K<1$ for $m \geqq N$ and for all $\lambda$ not real positive or zero.

(ii) $H^{1}=H^{0}+B A$ is an almost smooth perturbation of $H^{0}$. It will be shown below that these two assumptions insure the existence of the wave operators in the time independent form. With additional assumptions, one may also show that these operators coincide with

1) Actually T. Kato defines smoothness for more general operators than those considered in this paper. 
the wave operators defined in the time dependent manner. For this purpose we shall also assume:

(iii) For some $\lambda_{0}, \operatorname{Im}\left(\lambda_{0}\right) \neq 0$, the operator $|V|^{1 / 2} R^{0}\left(\lambda_{0}\right)$ is an integral operator with kernel in the Schmidt class.

Let $R^{p}(\lambda) \in \mathscr{B}(\mathscr{S})$ denote the resolvent operators corresponding to the operators $H^{p}, p=0,1$ (in formulas involving both $H^{0}, H^{1}$ we shall use $p, q=0,1, p+q=1)$.

LemMa 1 (T. Kato). Let $A, B$ be smooth with respect to $H^{0}$ and let (i) hold. Then for, $1 \in[0, \infty), \delta>0, p=0,1 R^{1}(1 \pm i \delta)$ is defined in terms of $R^{0}(1 \pm i \delta)$ by

$$
\begin{aligned}
R^{1}(1 \pm i \delta)= & R^{0}(1 \pm i \delta) \\
& -R^{0}(1 \pm i \delta) B(1+Q(1 \pm i \delta))^{-1} A R^{0}(1 \pm i \delta)
\end{aligned}
$$

where $Q(\lambda)=A R^{0}(\lambda) B$.

Proof. The proof is given in [2, p. 263]. Note that formula (2.1) is essentially the Neumann series for the resolvent since by assumption (i) $\sum_{v=0}^{\infty}(-1)^{v} Q^{v}$ is norm convergent and

$$
(1+Q)^{-1}=\sum_{v=0}^{\infty}(-1)^{v} Q^{v} .
$$

Lemma 2 (T. Kato). Let $A, B$ be smooth with respect to $H^{\circ}$ and let (i) hold. Then for $u \in \mathfrak{S}, 1 \in[0, \infty), \delta>0, p=0,1$ :

1. The vectors $A R^{p}(1 \pm i \delta) u, B R^{p}(1 \pm i \delta) u$ have limits along the reals $\delta \rightarrow 0$ in $\mathfrak{S}$.

2. If $A R^{p}(1 \pm i 0) u, B R^{p}(1 \pm i 0) u$ denote the limits $A R^{p}(1 \pm i 0) u=$ $\lim _{\dot{\delta} \rightarrow 0} A R^{p}(1 \pm i \delta) u, B R^{p}(1 \pm i 0) u=\lim _{\delta \rightarrow 0} B R^{p}(1 \pm i \delta) u$ then $A R^{p}(1 \pm$ i0) $u, B R^{p}(1 \pm i 0) u \in L_{2}\left([0, \infty): \mathfrak{S}_{2}\right)$.

3. If $Q=\sup _{\lambda, m}\left\|B R^{0}(\lambda) E^{0}\left(\Delta_{m}\right) A\right\|, \operatorname{Im}(\lambda) \neq 0, m \geqq N$, then

$$
\left\|A R^{1}(1 \pm i 0) u\right\| \leqq(1-\|Q\|)^{-1}\left\|A R^{0}(1 \pm i 0) u\right\|
$$

and

$$
\left\|B R^{1}(1 \pm i 0) u\right\| \leqq(1-\|Q\|)^{-1}\left\|B R^{0}(1 \pm i 0) u\right\| .
$$

Proof. [2, p. 264]. Note that in general the expression $R^{p}(1 \pm$ i0) $u$ does not make sense. The uniform bound $\|Q\|$ exists by the principle of uniform boundedness.

THEOREM 1. Let $A, B$ be smooth with respect to $H^{0}$ and let assumption (i) hold. Then the spectral measures $E^{p}(\Delta)$ corresponding to the operators $H^{p}, p=0,1$ are given by 
$(2.2)$

$$
\begin{aligned}
& \left(E^{p}(\Delta) u, v\right)=\left(E^{q}(\Delta) u, v\right) \\
+ & (-1)^{p} \frac{1}{\pi} \int_{\Delta}\left(\left\{R^{p}(1 \pm i 0) A B \operatorname{Im}\left(R^{q}(1 \pm i 0)\right)\right.\right. \\
+ & \left.\operatorname{Im}\left(R^{q}(1 \pm i 0) B A R^{p}(1 \mp i 0)\right\} u, v\right) d 1 \\
+ & \frac{1}{\pi} \int_{\Delta}\left(\left\{R^{p}(1 \pm i 0) A B \operatorname{Im}\left(R^{q}(1+i 0)\right) B A R^{p}(1 \mp i 0)\right\} u, v\right) d 1
\end{aligned}
$$

where $\operatorname{Im}\left(R^{p}(\lambda)\right)=(1 / 2 i)\left(R^{p}(\lambda)-R^{p}(\bar{\lambda})\right), u, v \in \mathfrak{S}, \Delta \subseteq[0, \infty)$.

Proof. Lemma 2 implies that the terms in the integrand are well defined. The integrands are absolutely integrable functions on $[0, \infty)$ because of the hypothesis that $A, B$ are smooth with respect to $H^{0}$.

To see this consider the integral

$$
\left.\int_{4} \mid R^{p}(1+i 0) B A R^{q}(1-i 0) u, v\right) \mid d 1 .
$$

By the Schwarz inequality (2.3) is dominated by

$$
\int_{\Delta}\left\|A R^{q}(1+i 0) u\right\|^{2} d 1 \int_{\Delta}\left\|B R^{p}(1-i 0) v\right\|^{2} d 1 .
$$

By Lemma 1,2 the product is less than

$$
p^{2}(1-\|Q\|)^{-1}\|u\|\|v\| \text {. }
$$

Therefore

$$
\int_{\Delta}\left|\left(R^{p}(1-i 0) B A R^{q}(1+i 0) u, v\right)\right| d 1 \leqq p^{2}(1-\|Q\|)^{-1}\|u\|\|v\|
$$

for all $\Delta \subseteq[0, \infty)$. The integral (2.3) corresponds to the first term in the integral on the right of (2.2). Bounds may be found for the integrals of the other terms of (2.2) similarly.

From the second resolvent equation $R^{1}(\lambda)=R^{0}(\lambda)-R^{0}(\lambda) B A R^{1}(\lambda)$ one may derive the identity

$$
\begin{aligned}
& \operatorname{Im}\left(R^{p}(1 \pm i \delta)\right)= \\
& \quad\left(1+(-1)^{p} R^{p}(1 \pm i \delta) A B\right) \operatorname{Im}\left(R ^ { q } ( 1 \pm i \delta ) \left(1+(-1)^{p} B A R^{p}(1 \mp i \delta)\right.\right.
\end{aligned}
$$

for all $1 \in[0, \infty), \delta>0$. Also it is known [2, p. 273] that

$$
\left(E^{p}(\Delta) u, v\right)=\lim _{\delta \rightarrow 0} \frac{1}{\pi} \int_{\Delta}\left(\operatorname{Im}\left(R^{p}(1 \pm i \delta)\right) u, v\right) d 1 .
$$

Employing (2.6), (2.7) and passing to the limit $\delta \rightarrow 0$ leads to (2.2). 
THeOREM 2. Let $A, B$ be smooth with respect to $H^{0}$ and let assumption (i) hold. Then the time independent wave operators $W_{ \pm}^{p}$, $p=0,1$ are determined by

$$
\left(W_{ \pm}^{p} u, v\right)=(u, v)+(-1)^{p} \frac{1}{\pi} \int_{0+}^{\infty}
$$

$$
\left(A \operatorname{Im}\left(R^{q}(1 \pm i 0)\right) u, B R^{p}(1 \pm i 0) v\right) d 1
$$

for $u, v \in \mathfrak{K}, p+q=1$.

Proof. By Lemma 2 the integrand in (2.8) is well defined and it is absolutely convergent by the hypothesis that $A, B$ are smooth with respect to $H^{0}$. It is proved in [2] that $W_{ \pm}^{p}$ are in general represented by

$$
\left(W_{ \pm}^{p} u, v\right)=(u, v)^{ \pm}(-1)^{p} \frac{1}{2 \pi i} \int_{-\infty}^{\infty}\left(A R^{q}(1 \pm i 0) u, B R^{p}(1 \pm i 0) v\right) d 1 .
$$

The integral appearing on the right side of (2.9) determines a bounded operator $X_{ \pm}^{p}$ and $\left(X_{ \pm}^{p} u, v\right)$ may be written

$$
\left(X_{ \pm}^{p} u, v\right)=\lim _{\delta \rightarrow 0}( \pm) \frac{1}{2 \pi i} \int_{-\infty}^{\infty}\left(R^{p}(1 \mp i \delta) B A R^{q}(1 \pm i \delta) u, v\right) d 1
$$

Since we have assumed that the spectrum of $H^{0}$ lies on $[0, \infty)$ it follows using Lemma 1 that $R^{p}(\lambda), p=0,1$ are regular in $\lambda$ if $\lambda$ is not real positive. Therefore the part of the integral (2.10) which is along the negative real axis may be deformed and

$$
\begin{array}{r}
\left(X_{ \pm}^{p} u, v\right)=\lim _{\delta \rightarrow 0}\left[( \pm) \frac{1}{2 \pi i} \int_{0+}^{\infty}\left(R^{p}(1 \mp i \delta) B A R^{q}(1 \pm i \delta) u, v\right) d 1\right. \\
\left.( \pm) \frac{1}{2 \pi i} \int_{0+}^{\infty}\left(R^{p}(1 \mp i \delta) B A R^{q}(1 \mp i \delta) u, v\right) d 1\right] \\
=\lim _{\delta \rightarrow 0} \frac{1}{\pi} \int_{0+}^{\infty}\left(R^{p}(1 \mp i \delta) B A \operatorname{Im}\left(R^{q}(1 \pm i \delta) u, v\right) d 1\right.
\end{array}
$$

Using (2.11) formula (2.9) reduces to (2.8).

COROLLARY. Then wave operators are also given by

$$
W_{ \pm}^{p}(u, v)=(u, v)+(-1) q \frac{1}{\pi} \int_{0+}^{\infty}
$$

$$
\left(A R^{q}(1 \pm i 0) u, B \operatorname{Im}\left(R^{p}(1 \pm i 0)\right) v\right) d 1
$$

for $u, v \in \mathfrak{K}, p+q=1$.

Proof. The proof is similar to the proof of the theorem. 
THEOREM 3. Let $A, B$ be almost smooth with respect to $H^{0}$ and let assumption (i) hold. Then:

1. The equation

$$
\left(W_{ \pm}^{p} u, v\right)=(u, v)+(-1)^{p} \frac{1}{\pi} \int_{0+}^{\infty}
$$

$$
\left(A \operatorname{Im}\left(R^{q}(1 \pm i o)\right) u, B R^{p}(1 \pm i 0) v\right) d 1
$$

defines operators $W_{ \pm}^{p} \in \mathscr{B}(\mathfrak{S}), p=0,1$ such that $W_{ \pm}^{p} W_{ \pm}^{q}=1$ and

$$
W_{ \pm}^{p} H^{q} W_{ \pm}^{q}=H^{p}, \quad p=0,1 .
$$

2. If in addition assumption (iii) holds then $W_{ \pm}^{p}$ coincide with the wave operators defined by the time dependent method.

Proof. Consider a sequence of operators $H_{n}^{0}$ where $H_{n}^{0}=H^{0} E^{0}\left(A_{n}\right)$, $\Delta_{n}=(1 / n, \infty), n \geqq 1$. Let $H_{n}^{1}$ be the sequence such that $H_{n}^{1}=H_{n}^{0}+$ $B \cdot A, n \geqq 1$. By hypothesis $A, B$ are smooth with respect to $H_{n}^{0}$ for all $n \geqq 1$. If $R_{n}^{p}(\lambda)=\left(H_{n}^{p}-\lambda I\right)^{-1}$ then by assumption (i) we may choose $N$ such that for $n \geqq N,\left\|B R_{n}^{0}(\lambda) A\right\| \leqq K<1$ for all $\lambda$ which are not real positive or zero. Applying Theorem 2 the wave operators $W_{ \pm}^{p}(n)$ corresponding to $H_{n}^{p}$ exist and are given in time independent form by

$$
\left(W_{ \pm}^{p}(n) u, v\right)=(u, v)+(-1)^{p} \frac{1}{\pi} \int_{0+}^{\infty}
$$

$$
\left(A \operatorname{Im}\left(R_{n}^{q}(1 \pm i 0)\right) u, B R_{n}^{p}(1 \pm i 0) v\right) d 1 .
$$

The operators $W_{ \pm}^{p}(n)$ are in $\mathscr{B}(\mathscr{S})$ and satisfy $W_{ \pm}^{p}(n) W_{ \pm}^{q}(n)=I$, $p+q=1$ and

$$
W_{ \pm}^{p}(n) R_{n}^{q}(\lambda) W_{ \pm}^{q}(n)=R_{ \pm}^{p}(\lambda)
$$

for all $\lambda, \operatorname{Im}(\lambda) \neq 0$. Also if $E_{n}^{p}(\Delta), \Delta \leqq[0, \infty)$ are the spectral measures of $H_{n}^{p}$ then $E_{n}^{p}(\Delta) W_{ \pm}^{p}(n)=W_{ \pm}^{p}(n) E_{n}^{q}(\Delta), \Delta \subseteq[0, \infty)$, which follows from (2.16). The operators $W_{ \pm}^{p}(n)$ agree with the wave operators defined in terms of $H_{n}^{p}$ in the time dependent manner [2, p. 271] and they satisfy $\left|\left(W_{ \pm}^{p}(n) u, v\right)\right| \leqq\left\|E^{p}\left(\Delta_{n}\right) v\right\|\left\|E^{q}\left(\Delta_{n}\right) u\right\|$. Formula (2.15) may be written

$$
\begin{aligned}
& \left(W_{ \pm}^{p}(n) u, v\right)=(u, v)+(-1)^{p} \int_{0+}^{\infty} \\
& \quad\left(A \operatorname{Im}\left(R^{q}(1 \pm i 0)\right) E^{q}\left(\Delta_{n}\right) u, B R^{p}(1 \pm i 0) E^{p}\left(\Delta_{n}\right) v\right) d 1 .
\end{aligned}
$$

Now consider the expression $Z_{n}(u, v)$ defined by

$$
Z_{n}(u, v)=\left(E^{q}\left(\Delta_{n}\right) u, v\right)+(-1)^{p} \frac{1}{\pi} \int_{1 / n+}^{\infty}
$$

$$
\left(A \operatorname{Im}\left(R^{q}(1 \pm i 0)\right) u, B R^{p}(1 \pm i 0) v\right) d 1 .
$$


To see that the integral in (2.18) is well defined write and

$$
R^{p}(\lambda)=S_{m}^{p}(\lambda)+R_{m}^{p}(\lambda) \text { where } R_{m}^{p}(\lambda)=R^{p}(\lambda) E^{p}\left(\Delta_{m}\right)
$$

$$
S_{m}^{p}(\lambda)=R^{p}(\lambda)\left(I-E^{p}\left(\Delta_{m}\right)\right)=\int_{0}^{1 / m}\left(1_{1}-\lambda\right)^{-1} d E^{p}\left(1_{1}\right) .
$$

The operator $S_{m}^{p}(\lambda)$ is regular in $\lambda$ for $\lambda \in(1 / n, \infty), m>n$. By hypothesis $A, B$ are smooth with respect to $H_{m}^{0}$. Applying Lemma 2,

$$
\begin{gathered}
\lim _{\delta \rightarrow 0} A R^{p}(1 \pm i \delta) u=A S_{m}^{p}(1) u+A R_{m}^{p}(1 \pm i 0) u \in \mathfrak{S}, \\
\lim _{\tilde{o} \rightarrow 0} B R^{p}(1 \pm i \delta) u=B S_{m}^{p}(1) u+B R_{m}^{p}(1 \pm i 0) u \in \mathfrak{K}, 1 \in(1 / n, \infty)
\end{gathered}
$$

and the integrals

$$
\begin{aligned}
& \int_{1 / n}^{\infty}\left\|A S_{m}^{q}(1) u\right\|^{2} d 1, \int_{1 / n}^{\infty}\left\|A R_{m}^{q}(1 \pm i o) u\right\|^{2} d 1, \\
& \int_{1 / n}^{\infty}\left\|B S_{m}^{p}(1) u\right\|^{2} d 1, \int_{1 / n}^{\infty}\left\|B R_{m}^{p}(1 \pm i 0) u\right\|^{2} d 1
\end{aligned}
$$

are convergent. Therefore the integral in (2.18) is absolutely convergent by the Schwarz inequality. Writing $Z_{n}(u, v)$ in the form

$$
\begin{aligned}
Z_{n}(u, v) & =\lim _{\delta \rightarrow 0} \frac{1}{\pi} \int_{1 / n^{+}}^{\infty} \\
& \left(\left(\left(1+(-1)^{p} R^{p}(1 \pm i \delta) B A\right) \operatorname{Im}\left(R^{q}(1 \pm i \delta)\right) u, v\right) d 1\right.
\end{aligned}
$$

by (2.5) and applying (2.6) one obtains

$$
\begin{aligned}
Z_{n}(u, v)= & \lim _{\delta \rightarrow 0} \int_{1 / n^{+}}^{\infty} \\
& \left(\left(1+(-1)^{q} B A R^{q}(1 \pm i \delta)\right) u, \operatorname{Im}\left(R^{p}(1 \mp i \delta)\right) v\right) d 1 .
\end{aligned}
$$

This implies, again by (2.5),

$$
\begin{aligned}
& Z_{n}(u, v)=\left(u, E^{p}\left(\Delta_{n}\right) v\right)+(-1)^{q} \frac{1}{\pi} \int_{1 / n^{+}}^{\infty} \\
&\left(B A R^{q}(1 \pm i 0) u, \operatorname{Im}\left(R^{p}(1 \mp i 0)\right) v\right) d 1
\end{aligned}
$$

Now because of the regularity of $S_{n}^{p}(1)$ for $1 \in(1 / n, \infty)$ and $R_{n}^{p}(1)$ for $1 \in(0,1 / n)$ we have $\operatorname{Im}\left(R_{n}^{p}(1 \pm i 0)\right) u=0$ for $1 \in(0,1 / n)$ and

$$
\operatorname{Im}\left(R_{n}^{p}(1 \pm i 0)\right) u=\operatorname{Im}\left(R^{p}(1 \pm i 0)\right) u,
$$

for $1 \in(1 / n, \infty)$. Using these relations it follows from (2.17), (2.18), (2.20) that $Z_{n}(u, v)=Z_{n}\left(E^{q}\left(\Delta_{n}\right) u, v\right)=Z_{n}\left(u, E^{p}\left(\Delta_{n}\right) v\right)$ and also

$$
Z_{n}(u, v)=\left(W_{ \pm}^{p}(n) E^{q}\left(\Delta_{n}\right) u, E^{p}\left(\Delta_{n}\right) v\right)=\left(W_{ \pm}^{p}(n) u, v\right) .
$$


Since

$$
Z_{n}(u, v)=\left(W_{ \pm}^{p}(n) u, v\right),\left|Z_{n}(u, v)\right| \leqq\left\|E^{p}\left(\Delta_{n}\right) v\right\|\left\|E^{q}\left(\Delta_{n}\right) u\right\|,
$$

the sequence $Z_{n}(u, v)$ converges to the right side of (2.13) and defines an operator $W_{ \pm}^{p} \in \mathscr{S}(\mathfrak{C})$. The operators $R_{n}^{p}(\lambda)$ converge strongly to $R^{p}(\lambda), \operatorname{Im}(\lambda) \neq 0$, the operators $W_{ \pm}^{p}(n)$ converge weakly to $W_{ \pm}^{p}, n \rightarrow \infty$, and $W_{ \pm}^{p}(n) W_{ \pm}^{p}=I, W_{ \pm}^{p}(n) R_{n}^{q}(\lambda) W_{ \pm}^{q}(n)=R_{n}^{p}(\lambda), n \geqq 1$. From these relations it follows that the first part of the conclusion of the theorem is valid.

If the hypothesis of part 2 of the theorem holds then the wave operators corresponding to $H^{p}$ exist as defined in the time dependent manner [1, p. 546]. Let us denote these wave operators by $\hat{W}_{ \pm}^{p}$. It is easily seen that $W_{ \pm}^{p}(n)$ converge strongly to $\hat{W}_{ \pm}^{p}$. Since $W_{ \pm}^{p}(n)$ also converge weakly to $W_{ \pm}^{p}$ it must be that $W_{ \pm}^{p}=\hat{W}_{ \pm}^{p}$ and the operators defined in the two different ways coincide.

COROLLARY. The conclusion of Theorem 1 holds if the assumption that $H^{1}$ is a smooth perturbation of $H^{0}$ is replaced by the assumption that $H^{1}$ is an almost smooth perturbation of $H^{0}$.

The proof proceeds along the same lines as the proof of the theorem.

3. Application to ordinary differential operators. To apply Theorem 3 consider a self-adjoint operator $H^{0}$ on $L_{2}(-\infty, \infty)$ which is determined by the formal ordinary differential operator

$$
L_{0}=(-1)^{v}(d / d x)^{n}, n=2 v,
$$

defined on $(-\infty, \infty)$. The resolvent $R^{0}(\lambda)=\left(H^{0}-\lambda I\right)^{-1}$ may be explicitly calculated. Let $\lambda, w$ be complex variables defined by

$$
\lambda=r e^{i \theta}, w=r^{1 / n} \exp (i \theta / n), r \geqq 0,0 \leqq \theta<2 \pi,
$$

and define functions $s_{j}^{0}(x, \lambda)=\exp \left(e_{j} w x\right), j=1, \cdots, n$ where $e_{j}, j=$ $1, \cdots, n$ are complex roots of unity with increasing argument

$$
-\frac{\pi}{2} \leqq \arg e_{1}<\cdots<\arg e_{n}<\frac{3 \pi}{2} .
$$

The functions $s_{j}^{0}(x, \lambda), j=1, \cdots, n$ form a fundamental set of solutions of the equation $L_{0} y=\lambda y,-\infty<x<\infty$. The resolvent $R^{0}(\lambda)$ is an integral operator whose kernel is the Green's function

$$
G^{0}(x, y: \lambda)=\frac{1}{n w^{n-1}} \sum_{k=v+1}^{n} e_{k} s_{k}^{0}(x, \lambda) s_{k-v}^{0}(y, \lambda)
$$


for $x \geqq y$ with variables $x, y$ interchanged if $x<y$. The kernel of the spectral measure $E^{0}(\Delta)$ associated with $H^{0}$ is given by

$$
\mathscr{E}^{0}(x, y: \Delta)=\lim _{\delta \rightarrow 0} \frac{1}{\pi} \int_{\Delta} \operatorname{Im}\left(G^{0}(x, y: 1+i \delta) d 1 .\right.
$$

More precisely and explicitly with $\Delta_{1}=(\Delta)^{1 / n}, s^{n}=1$

$$
\begin{aligned}
E^{0}(\Delta) u= & \frac{1}{2 \pi i} \int_{\Delta_{1}} \sum_{k=v+1}^{n} \int_{-\infty}^{\infty} \\
& \left\{e_{k} \exp \left[e_{k} s(x-y)\right]-\bar{e}_{k} \exp \left[\bar{e}_{k} s(x-y)\right]\right\} u(y) d y d s
\end{aligned}
$$

for $u \in L_{2}(-\infty, \infty), \Delta \subseteq[0, \infty)$. Suppose that $A, B$ are real multiplication operators $A u=f_{2}(x) u, B u=f_{1}(x) u$, where

$$
f_{1}, f_{2} \in L_{2}(-\infty, \infty), f_{1}, f_{2} \in C(-\infty, \infty) .
$$

Then the differential operator $L^{1}=L^{0}+\left(f_{1}(x) f_{2}(x)\right)$ determines a selfadjoint operator $H^{1}=H^{0}+B \cdot A$ on $L_{2}(-\infty, \infty)$. To show $H^{1}$ is almost $H^{0}$ smooth, but not $H^{0}$ smooth we must show

$$
\int_{0}^{\infty} \int_{-\infty}^{\infty}\left|f_{i}(x)\left(R^{0}(1 \pm i \delta) E^{0}\left(\Delta_{m}\right) u\right)(x)\right|^{2} d x d 1 \leqq P\left(\Delta_{m}\right)\|u\|, \quad i=1,2
$$

where $\Delta_{m}$ is any interval $\Delta_{m}=(1 / m, \infty)$. Since

$$
R_{m}^{0}(\lambda)=R^{0}(\lambda) E^{0}\left(\Delta_{m}\right)=\int_{1 / m}^{\infty}\left(1_{1}-\lambda\right)^{-1} d E^{0}\left(1_{1}\right)
$$

is regular for $\lambda$ real $0 \leqq \lambda \leqq 1 / 2 m$ equation (3.4) follows if

$$
\begin{aligned}
& \int_{1 / 2 m}^{\infty} \int_{-\infty}^{\infty}\left|f_{i}(x) \int_{-\infty}^{\infty} G^{0}(x, y: 1 \pm i \delta) u(y) d y\right|^{2} d x d 1 \leqq P_{1}\left(\Delta_{m}\right)\|u\|, \\
& i=1,2
\end{aligned}
$$

for all $m \geqq 1$. Employing (3.2) it turns out (3.5) holds with

$$
P_{1}\left(\Delta_{m}\right)=(2 m)^{2} n\left|\operatorname{Re} e_{v+2}\right|^{-1} \pi \max _{i=1,2}\left(\int_{-\infty}^{\infty} f_{i}^{2} d x\right)
$$

and (3.4) with

$$
P\left(\Delta_{m}\right)=P_{1}\left(\Delta_{m}\right)+\max _{i=1,2}\left(\int_{-\infty}^{\infty} f_{i}^{2} d x\right) .
$$

Now assume that the functions $f_{1}, f_{2}$ satisfy the following conditions:

(a) $f_{i}(x), g_{i}(m: x) \in C(-\infty, \infty) \cap L_{1}(-\infty, \infty) \cap L_{2}(-\infty, \infty)$ and

$$
\int_{-\infty}^{\infty}\left|f_{1}(x)\right|^{2} d x\left(\int_{-\infty}^{\infty}\left|f_{n+1}(x)\right| d x\right)^{2}<1
$$

where 


$$
\begin{gathered}
f_{i+1}=\int_{x}^{\infty} f_{i} d x, g_{i+1}=\int_{x}^{\infty} g_{i} d x, \quad i=3, \cdots, n, \\
g_{2}(m: x)=E^{0}\left(\Delta_{m}\right) f_{2} u, f_{3}=\left(\int_{x}^{\infty} f_{2}^{2} d x\right)^{\frac{1}{2}}, g_{3}=\left(\int_{x}^{\infty} g_{2}^{2} d x\right)^{\frac{1}{2}},
\end{gathered}
$$

for any $u \in C^{\infty}(-\infty, \infty),\|u\|<1$,

(b) $\int_{-\infty}^{\infty} \int_{-\infty}^{\infty}\left|f_{1}(x) f_{2}(y) G^{0}\left(x, y: \lambda_{0}\right)\right|^{2} d x d y<\infty$

for some $\lambda_{0}, \operatorname{Im}\left(\lambda_{0}\right) \neq 0$. Assumption (i) is valid if there exists $N$ such that

$$
\int_{-\infty}^{\infty}\left|f_{1}(x) \int_{-\infty}^{\infty} G^{0}(x, y: \lambda) g_{2}(m: y) d y\right|^{2} \quad d x \leqq K<1
$$

for $m \geqq N$ and all $\lambda$ not real positive or zero. Integrating by parts $(n-1)$-times $(3.6)$ is equivalent to

$$
\int_{-\infty}^{\infty}\left|f_{1}(x) \int_{-\infty}^{\infty}\left(\frac{\partial^{n-1} G^{0}}{\partial y^{n-1}}\right) g_{n+1}(m: y) d y\right|^{2} \quad d x \leqq K<1
$$

Inspection of (3.2) shows $\left|\partial^{n-1} G^{0} / \partial y^{n-1}\right| \leqq 1$ for all $x, y$ and $\lambda$ not positive real. Therefore (3.7) holds if

$$
\int_{-\infty}^{\infty}\left|f_{1}(x)\right|^{2} d x\left(\int_{-\infty}^{\infty}\left|g_{n+1}(m: y)\right| d y\right)^{2} \leqq K<1
$$

Since $E^{0}\left(\Delta_{m}\right) f_{2} u=g_{2}(m: x)$ converges to $f_{2}(x) u, n \rightarrow \infty$, in the $L_{2}(-\infty, \infty)$ norm it follows, using $(a)$, that there exists $N$ such that (3.8) holds for $m \geqq N$. The assumption (b) implies that the operator $|V|^{1 / 2} R^{0}\left(\lambda_{0}\right)$ has a kernel in the Schmidt class. Therefore when (a), (b) hold the wave operators exist corresponding to $L^{0}, L^{1}$, as a consequence of Theorem 3.

Similar results to those stated above apply to the differential operator $L^{0}=-(d / d x)^{2}$ defined on $[0, \infty)$ with the boundary condition $u^{\prime}(0)=0$ imposed at $x=0$. In this case we assume that $f_{1}, f_{2}$ are such that

$$
f_{1}, f_{2}, f_{3}, g_{2}, g_{3}
$$

are in $C(0, \infty) \cap L_{1}(0, \infty) \cap L_{2}(0, \infty)$ and

$$
\int_{0}^{\infty} f_{1}^{2} d x\left(\int_{0}^{\infty}\left|f_{3}\right| d x\right)^{2}<1
$$

Again $L^{1}=L^{0}+\left(f_{2}(x) \cdot f_{1}(x)\right)$ is almost smooth but not smooth with respect to $L^{0}[3$, p. 381]. 


\section{REFERENCES}

1. T. Kato, Perturbation theory for linear operators, Springer Verlag, Berlin, 1966.

2. - Wave operators and similarity for some non-self-adjoint operators, Math Ann. 169 (1966), 258-279.

3. J. Moser, Störungstheorie des kontinuierlichen Spektrums für gewöhnliche Differentialgleichungen zweiter Ordnung, Math. Ann. 125 (1953), 366-393.

Received November 19, 1969.

Middle East Technical University, AnKara 


\section{PACIFIC JOURNAL OF MATHEMATICS}

\section{EDITORS}

\author{
H. SAMELSON \\ Stanford University \\ Stanford, California 94305

\section{Richard Pierce} \\ University of Washington \\ Seattle, Washington 98105
}

J. DugundJI

Department of Mathematics

University of Southern California

Los Angeles, California 90007

RichaRd ARENS

University of California

Los Angeles, California 90024

\section{ASSOCIATE EDITORS}
E. F. BECKENBACH
B. H. NeUMANN
F. WOLE
K. YoshidA

\section{SUPPORTING INSTITUTIONS}

\author{
UNIVERSITY OF BRITISH COLUMBIA \\ CALIFORNIA INSTITUTE OF TECHNOLOGY \\ UNIVERSITY OF CALIFORNIA \\ MONTANA STATE UNIVERSITY \\ UNIVERSITY OF NEVADA \\ NEW MEXICO STATE UNIVERSITY \\ OREGON STATE UNIVERSITY \\ UNIVERSITY OF OREGON \\ OSAKA UNIVERSITY \\ UNIVERSITY OF SOUTHERN CALIFORNIA
}

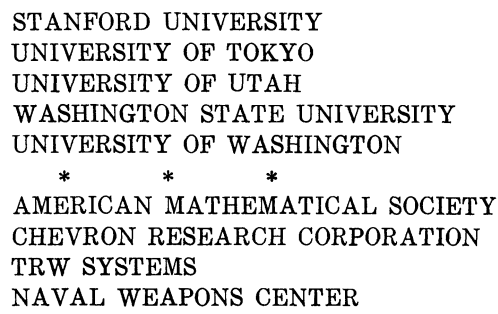

The Supporting Institutions listed above contribute to the cost of publication of this Journal, but they are not owners or publishers and have no responsibility for its content or policies.

Mathematical papers intended for publication in the Pacific Journal of Mathematics should be in typed form or offset-reproduced, (not dittoed), double spaced with large margins. Underline Greek letters in red, German in green, and script in blue. The first paragraph or two must be capable of being used separately as a synopsis of the entire paper. The editorial "we" must not be used in the synopsis, and items of the bibliography should not be cited there unless absolutely necessary, in which case they must be identified by author and Journal, rather than by item number. Manuscripts, in duplicate if possible, may be sent to any one of the four editors. Please classify according to the scheme of Math. Rev. Index to Vol. 39. All other communications to the editors should be addressed to the managing editor, Richard Arens, University of California, Los Angeles, California, 90024.

50 reprints are provided free for each article; additional copies may be obtained at cost in multiples of 50 .

The Pacific Journal of Mathematics is published monthly. Effective with Volume 16 the price per volume (3 numbers) is $\$ 8.00$; single issues, $\$ 3.00$. Special price for current issues to individual faculty members of supporting institutions and to individual members of the American Mathematical Society: $\$ 4.00$ per volume; single issues $\$ 1.50$. Back numbers are available.

Subscriptions, orders for back numbers, and changes of address should be sent to Pacific Journal of Mathematics, 103 Highland Boulevard, Berkeley, California, 94708.

PUBLISHED BY PACIFIC JOURNAL OF MATHEMATICS, A NON-PROFIT CORPORATION

Printed at Kokusai Bunken Insatsusha (International Academic Printing Co., Ltd.), 7-17, Fuj̣imi 2-chome, Chiyoda-ku, Tokyo, Japan. 


\section{Pacific Journal of Mathematics}

\section{Vol. 35, No. $2 \quad$ October, 1970}

Valentin Danilovich Belousov and Palaniappan L. Kannappan, Generalized Bol functional equation .................................... 259

Charles Morgan Biles, Gelfand and Wallman-type compactifications ........... 267

Louis Harvey Blake, A generalization of martingales and two consequent convergence theorems .................................... 279

Dennis K. Burke, On p-spaces and $w \Delta$-spaces..................... 285

John Ben Butler, Jr., Almost smooth perturbations of self-adjoint operators . . . . . . 297

Michael James Cambern, Isomorphisms of $C_{0}(Y)$ onto $C(X) \ldots \ldots \ldots \ldots \ldots . \ldots 307$

David Edwin Cook, A conditionally compact point set with noncompact closure ... 313

Timothy Edwin Cramer, Countable Boolean algebras as subalgebras and homomorphs .........................................

John R. Edwards and Stanley G. Wayment, A v-integral representation for linear operators on spaces of continuous functions with values in topological vector spaces.............................................

Mary Rodriguez Embry, Similarities involving normal operators on Hilbert

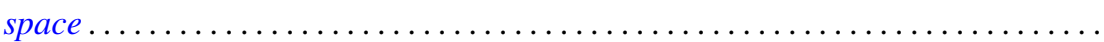

Lynn Harry Erbe, Oscillation theorems for second order linear differential

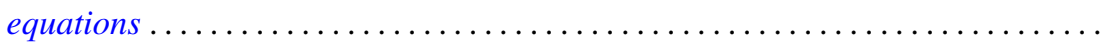

William James Firey, Local behaviour of area functions of convex bodies .......... Joe Wayne Fisher, The primary decomposition theory for modules ..............

Gerald Seymour Garfinkel, Generic splitting algebras for Pic ..................

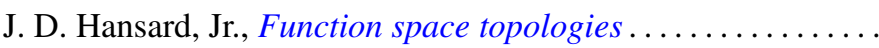

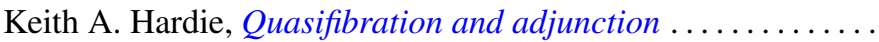

G. Hochschild, Coverings of pro-affine algebraic groups ...........

Gerald L. Itzkowitz, On nets of contractive maps in uniform spaces ..

381

389

399

417

Melven Robert Krom and Myren Laurance Krom, Groups with free nonabelian subgroups....................................

James Robert Kuttler, Upper and lower bounds for eigenvalues by finite differences ......................................

Dany Leviatan, A new approach to representation theory for convolution transforms . . .

Richard Beech Mansfield, Perfect subsets of definable sets of real numbers ...

Brenda MacGibbon, A necessary and sufficient condition for the embedding of a

Lindelof space in a Hausdorff $\mathscr{H} \sigma$ space ..................

David G. Mead and B. D. McLemore, Ritt's question on the Wronskian ....

Edward Yoshio Mikami, Focal points in a control problem .....

Paul G. Miller, Characterizing the distributions of three independent n-dimensional random variables, $X_{1}, X_{2}, X_{3}$, having analytic characteristic functions by the joint distribution of $\left(X_{1}+X_{3}, X_{2}+X_{3}\right)$. . .

P. Rosenthal, On the Bergman integral operator for an elliptic partial differential equation with a singular coefficient....

Douglas B. Smith, On the number of finitely generated $O$-group 